\title{
Evaluación de la calidad docente y promoción del profesorado (VI). Legislación universitaria española (d): modificación de la Ley Orgánica de Universidades. Profesorado funcionario: de la habilitación a la acreditación (2004-2008) (1. ${ }^{\mathrm{a}}$ parte)
}

\author{
J. Carreras
}

Después de ganar el Partido Socialista las elecciones generales de marzo de 2004, el nuevo Gobierno surgido de éstas inició un proceso de modificación de la Ley Orgánica de Universidades (LOU) promulgada el año 2002 por el gobierno del Partido Popular, dirigido a potenciar la autonomía de las universidades. En este artículo se explica el desarrollo del proceso de tramitación parlamentaria de la nueva ley (Ley Orgánica de modificación de la LOU) hasta su promulgación en abril de 2007; se comentan las modificaciones introducidas en el articulado referente al profesorado universitario funcionario y se analiza la subsiguiente normativa de desarrollo reglamentario. La nueva legislación redujo a dos los cuerpos de funcionarios docentes universitarios: el cuerpo de catedráticos de universidad y el cuerpo de profesores titulares de universidad. Para ambos, el procedimiento de incorporación del profesorado se estructuró en dos fases: una fase de acreditación nacional y una fase de concurso para el acceso a plazas concretas convocadas por las universidades. El proceso de acreditación nacional tendría por finalidad valorar los méritos y las competencias de los aspirantes para garantizar la calidad del profesorado funcionario. Se llevaría a cabo mediante el examen de la documentación presentada por los solicitantes, por comisiones específicas designadas por el Consejo de Universidades. El procedimiento de los concursos para el acceso a plazas de los cuerpos docentes lo regularían los estatutos de cada universidad, siguiendo unas condiciones mínimas establecidas por la nueva Ley.

Palabras clave. Legislación universitaria española, acreditación. Selección del profesorado universitario.

Evaluation of teaching and faculty promotion (VI). University Spanish legislation (d): modification of the Organic Law of Universities. Appointment for the bodies of functionary professors: from the system of 'habilitation' to the system of 'accreditation' (2004-2008) (1st part)

The new Spanish Government formed by the Socialist Party in the year 2004, in order to increase the autonomy of the universities, initiated the process of modification of the organic Law of Universities (LOU) established in 1983 by the previous Government constituted by the Popular Party. In this article we describe the process developed by the Spanish parliament until the promulgation of a new law (Organic Law of Modification of the LOU) in April of 2007. We comment the modifications introduced in the LOU that effected the categories of functionary professors, and we analyze the norms subsequently established. The categories of university functionary professors were reduced to two ('catedráticos' and 'profesores titulares'), and for both categories was established a procedure of appointment with two steps: a first step of national 'accreditation' and a second step of concourse to the specific positions convoked by the universities. The objective of the process of national 'accreditation' would be to evaluate the merits and competencies of the candidates in order to guarantee the quality of the professoriate. It would be carried out by specific commissions nominated by the University Council. The rules of the specific concourses developed by the universities would be established by their statutes.

Key words. Appointment of the university professors. University Spanish Legislation.

\section{Introducción}

Como mencionamos en el artículo anterior de esta serie [1], tras la victoria del Partido Socialista Obrero Español (PSOE) en las elecciones
Departamento de Ciencias Fisiológicas I. Facultad de Medicina. Universitat de Barcelona. Barcelona, España.

Correspondencia: Dr. Josep Carreras. Departamento de Ciencias Fisiológicas I. Facultad de Medicina. Universitat de Barcelona. Casanova, 143. E-08036 Barcelona.

Fax: +34934035882

E-mail

jcarreras@ub.edu 
generales celebradas en marzo de 2004, el nuevo presidente del gobierno, José Luis Rodríguez Zapatero, en su discurso de investidura reiteró el compromiso de reformar la Ley de Ordenación Universitaria (LOU). En la primera parte de este artículo describimos el proceso de elaboración del proyecto de la Ley Orgánica de Modificación de la LOU (LOMLOU) y su tramitación parlamentaria hasta la aprobación por las Cortes en abril de 2007. En la segunda parte, comentaremos las modificaciones relativas al profesorado funcionario introducidas por la Ley, y finalizaremos con el análisis de la normativa de desarrollo reglamentario de ésta.

\section{Elaboración y tramitación parlamentaria del proyecto de ley de modificación de la LOU}

La intención del gobierno socialista de iniciar rápidamente la modificación de la LOU recibió de inmediato la oferta de apoyo y colaboración por parte de la Conferencia de Rectores de Universidades Españolas (CRUE) [2]. En la asamblea general de dicho organismo, celebrada a finales de abril de 2004, tanto el Secretario de Estado de Universidades como el presidente de la Conferencia afirmaron que las modificaciones de la LOU se realizarían a dos velocidades y a través de aspectos puntuales, y coincidieron en señalar que la modificación más urgente se centraba en el sistema de selección del profesorado. A este respecto, el Secretario de Estado recordó que el programa electoral del PSOE planteaba la posibilidad de crear un sistema de acreditación que, en su opinión, habría 'que definir entre todos para garantizar que nadie que no tenga las condiciones adecuadas se pueda presentar a las comisiones de adscripción' [3]. Por otra parte, la CRUE se comprometió a elaborar un documento con sus propuestas sobre política universitaria para negociar con el Ministerio [4].

La ministra de educación y ciencia, María Jesús San Segundo, en sus primeras comparecencias ante las Comisiones de Educación y Ciencia del Congreso de los Diputados y del Senado, en las que expuso las líneas generales de la actuación de su departamento, señaló, entre los aspectos más conflictivos de la LOU, que debían determinar las modificaciones oportunas, los costes humanos y económicos que suponían las habilitaciones y las reclamaciones y recursos que se planteaban continuamente ante los informes de la Agencia Nacional de Evaluación de la Calidad y Acreditación (ANECA), y precisó: 'La habilitación se ha revelado costosa e ineficaz como reflejan los informes de la Conferencia de Rectores. En las cuatro primeras convocatorias, los tribunales han requerido la participación de entre 500 y 700 profesores permanentes para seleccionar a 200 o 250 habilitados. Los costes y la frustración generados en convocatorias muy concurridas, especialmente para las plazas de profesor titular, han sido muy negativas para la incentivación de la carrera profesional. Es necesario retomar el consenso existente acerca del diseño de procedimientos de acreditación nacional que garanticen la calidad de los candidatos y que agilicen los procesos de cobertura de plazas permanentes con los profesores de la mayor valía' $[5,6]$.

En cumplimiento de su compromiso, la CRUE, partiendo de las conclusiones elaboradas un mes antes por la Comisión Académica Sectorial de las Universidades Españolas, CASUE [7], aprobó a finales de junio un documento en el que exponía su posición respecto a las orientaciones de la política universitaria y a los aspectos susceptibles de ser modificados en la LOU [8]. Por lo que se refiere al sistema de habilitación, el documento señaló la necesidad de definir, en un plazo que no debía extenderse más allá del mes de octubre y con la participación de la CRUE, un sistema alternativo que se complementaría con el proceso propiamente competitivo que, mediante el correspondiente concurso de acceso, desarrollase cada universidad.

En su primera reunión con el pleno del Consejo de Coordinación Universitaria (CCU), celebrado el mes de julio, la ministra adelantó las líneas de actuación del Gobierno por lo que respecta a la modificación del sistema de habilitación [9]. Precisó que en el mes de octubre se propondría al Consejo un modelo de acceso a la función pública docente estructurado en dos etapas: acreditación y concurso. Para la primera se fijaría un proceso general de evaluación del historial académico, investigador y docente de todos los candidatos que lo solicitaran, con criterios claros y objetivos. La acreditación la realizarían comisiones constituidas por evaluadores de trayectoria reconocida en su área, procedentes de las distintas comunidades autónomas, y de carácter rotatorio. A la modifica- 
ción de la LOU en el Parlamento deberían seguir desarrollos normativos que perfilasen los procedimientos que habría que utilizar en las fases de acreditación y de concurso. Con todo ello, se dibujaba un horizonte de reformas que ocuparían entorno a un año y medio.

Transcurrido el paréntesis estival, a mitad de octubre, el presidente del gobierno se reunió en la Moncloa con los rectores de las universidades. Tras el encuentro, el presidente de la CRUE manifestó que Zapatero había reiterado su intención de reformar la LOU, 'cambiando la legislación desde el diálogo y el consenso, sin alterar la vida académica'. La reforma incluiría 'un incremento de la autonomía de las universidades y la creación de un nuevo sistema de selección del profesorado por méritos' $[10,11]$. Dos meses después, el Ministerio de Educación y Ciencia (MEC) presentó a la CRUE y al CCU, reunidos en Valencia los días 15 y 16 de diciembre, las propuestas del Gobierno relativas a la reforma de la LOU $[12,13]$. En cuanto a la selección del profesorado funcionario, éstas suponían sustituir el proceso de habilitación por otro de 'acreditación' que valoraría los méritos y las competencias de los aspirantes. Con el fin de garantizar la transparencia del proceso, comisiones específicas para los distintos campos, constituidas por catedráticos con tres sexenios, elegidos por sorteo, propondrían los criterios objetivos que deberían cumplir los candidatos. Dichos criterios, que deberían ser aprobados por el CCU, tendrían habitualmente una validez de varios años. Periódicamente, comisiones de acreditación, que se renovarían cada dos años, evaluarían las solicitudes presentadas, y aplicarían estos criterios. En una fase posterior, las universidades escogerían a los mejores candidatos entre el colectivo de acreditados en un área de conocimiento, mediante concursos de acceso evaluados por comisiones constituidas por profesores que reuniesen las condiciones fijadas por la legislación. La CRUE, la Federación de Enseñanza de CC.OO. y la UGT aplaudieron las medidas propuestas por el Gobierno [14]. En cambio, el Partido Popular (PP) las criticó y afirmó que promovían una selección del profesorado 'sin garantías' y 'opaca', que dejaría 'inerme a la comunidad universitaria contra los riesgos de arbitrariedad y favoritismos que podrían producirse' [15].

A comienzos de marzo del año 2005 el presidente de la CRUE manifestó que el borrador de la ley de reforma de la LOU estaría listo en po- cas semanas [16], pero transcurrió el verano sin que así fuera. Por este motivo, iniciado el mes de septiembre, la Asamblea General de este organismo manifestó su inquietud y preocupación y requirió un calendario definido que concretara los plazos del debate y la modificación de la Ley [17]. Finalmente, el 27 de octubre la ministra de Educación y Ciencia presentó al CCU el borrador del proyecto de reforma de la LOU elaborado por el Gobierno [18,19]. En una reunión celebrada el mismo día, la Asamblea General de la CRUE acordó iniciar de inmediato el estudio y la valoración del texto y organizó un proceso de debate y análisis a través de sus comisiones sectoriales y de cuatro grupos específicos constituidos a tal afecto [20]. Las conclusiones derivadas de estos estudios, así como las propuestas realizadas por las universidades, colectivos universitarios y otras instituciones, fueron presentados a la siguiente Asamblea General celebrada el 14 de diciembre [21]; en dicha asamblea se debatió y aprobó el documento relativo a profesorado y asuntos académicos, elaborado por la CASUE un mes antes [22]. Una nueva Asamblea General de la CRUE, que tuvo lugar el 25 de enero del año 2006 [23], discutió los documentos elaborados por los otros grupos de trabajo y aprobó, por amplio consenso, los textos definitivos [24], que fueron entregados al Ministerio al día siguiente [25].

Entre tanto, se habían manifestado sobre el borrador del proyecto ministerial los gobiernos de Cataluña y del País Vasco, que lo habían calificado de centralista y excesivamente reglamentario, y aseguraban que invadía competencias de las autonomías en diversos aspectos, entre otros la selección del profesorado. También lo había criticado el director general de Universidades del Gobierno Navarro, quien calificó como 'un error' la sustitución del sistema de habilitación por el de acreditación sin cupos propuesto por el Gobierno $[26,27]$. Pilar del Castillo, ex ministra de Educación, Cultura y Deporte del anterior Gobierno del PP, había dicho que el Gobierno presentaba una 'reforma marginal', que no 'se correspondía con ninguna de las reivindicaciones expresadas por el PSOE durante la tramitación de la LOU' [28].

$\mathrm{El}$ análisis del borrador realizado por las organizaciones sindicales -FETE-UGT, Federación de Enseñanza de Comisiones Obreras y Sindicato Independiente de Personal de Universidad (SIPU)- fue especialmente crítico y se concretó en la presentación de numerosas enmiendas [29- 
34]. La Federación de Asociaciones de Catedráticos de Universidad opinó que el proyecto suponía un 'importante retroceso' para el sistema universitario español y propiciaba una 'vuelta al pasado' en, por lo menos, dos aspectos: la elección del rector y el acceso al profesorado $[35,36]$. La Plataforma Estatal de Profesores Titulares de Escuelas Universitarias, que integraba más de 10.000 docentes, se mostró 'indignada' y manifestó su 'enérgico rechazo' a las disposiciones previstas referentes a la extinción de dicho cuerpo docente; amenazó, incluso, con ir a la huelga de aprobarse el texto tal como se había presentado [37-39]. Y la Plataforma Estatal de Profesores Contratados de Universidad hizo una valoración muy negativa del borrador por cuanto 'empeoraba, más si cabe, su situación', presentando las correspondientes modificaciones [40]. Por otra parte, la Asociación para el Avance de la Ciencia y la Tecnología en España (AACTE) dio a conocer sus reservas sobre la repercusión que para la calidad del profesorado pudieran tener las propuestas ministeriales [41], y el director y el Consejo Asesor de la ANECA criticaron diversos aspectos del proyecto referentes, principalmente, al profesorado y a la propia Agencia [42-44].

Parte de estas críticas fue contestada por representantes del MEC. La ministra San Segundo, en declaraciones a la prensa, manifestó su confianza en que el dictamen del Consejo de Estado sobre el borrador ministerial confirmaría que el reparto de competencias propuesto en el mismo era 'acertado', en contra de las críticas expresadas por ciertos gobiernos autonómicos [45]. Y el secretario de Estado de Universidades, que había comentado que el proyecto de ley no 'era de su gusto' [46], precisó en una reunión de la Comisión Sectorial de la Conferencia de Rectores que la propuesta no era 'un documento cerrado' y dejó clara su voluntad de negociar la reforma de la LOU con los sindicatos [47]. Sin embargo, el día 7 de abril fue nombrada nueva ministra de Educación y Ciencia, Mercedes Cabrera, la cual hasta entonces había ocupado la presidencia de la Comisión de Educación y Ciencia del Congreso de los Diputados [48].

En el acto de toma de posesión, la nueva ministra dijo que frente al desafío que representaba la incorporación de la universidad española al EEES haría todo lo posible para que la reforma de la misma 'no fuera traumática sino todo lo contrario' [49], y en su primera reunión con la Conferencia de Rectores anunció que el Gobierno aprobaría una nueva versión del anteproyecto de ley de modificación de la LOU en mayo [50]. Cumpliendo este anuncio, a finales de dicho mes, en su comparecencia ante la Comisión de Educación y Ciencia del Congreso de los Diputados [51,52], en la que expuso las líneas prioritarias de actuación de su departamento, Mercedes Cabrera dio a conocer el borrador del anteproyecto de ley preparado por el Ministerio [53] y anunció su inminente remisión al CCU. A mitad de junio, en su comparecencia ante la Comisión de Educación y Ciencia del Senado, explicó el estado en que se hallaba el proceso de modificación de la LOU [54].

En la Asamblea General celebrada a finales de mayo, la CRUE debatió el borrador del anteproyecto de ley y lo valoró de forma positiva, si bien decidió trasladar al Ministerio ciertas observaciones sobre algunas enmiendas de la Conferencia al primer borrador que no habían sido recogidas y sobre determinados aspectos novedosos del nuevo borrador [55]. También presentó sus enmiendas al documento la FETE-UGT [56], mientras que el PP criticó muy duramente la propuesta ministerial [57]. Por último, el día 30 de ese mes, el Consejo de Ministros aprobó el texto del anteproyecto de Ley Orgánica por el que se modificaba la LOU y lo remitió a la CRUE y al Consejo de Estado para su informe preceptivo [58-60]. La Asamblea General de la CRUE analizó el texto con fecha 5 de julio y, al verificar que buena parte de sus enmiendas no habían sido tenidas en consideración, decidió reiterarlas y mantener sus posiciones al respecto [61]. El Consejo de Estado emitió su informe el día 9 de julio [62] y el Consejo de Ministros, con fecha 1 de septiembre, aprobó, para remitirlo a las Cortes, el texto definitivo del Proyecto de Ley, que recogía las mejoras de carácter técnico realizadas por el Consejo de Estado [63-65].

Las reacciones a dicho Proyecto no se hicieron esperar. Por lo que respecta a los partidos políticos, las más negativas fueron las del PP, cuyos representantes insistieron en que 'con el nuevo sistema de selección del profesorado, la universidad española se podía convertir en la más cerrada, endogámica y localista de toda la Unión Europea, y no se garantizaba 'la igualdad de acceso a la función docente' [66-68], y las de Esquerra Republicana de Catalunya , que manifestó que la reforma se había elaborado 'sin tener en cuenta el criterio de las comunidades autónomas' [69]. Menos crí- 
tica, Izquierda Unida difundió un comunicado en el que señalaba que los cambios propuestos eran 'poco ambiciosos' y que la reforma era 'tímida' [68], y la Federación de Enseñanza de CC.OO., si bien manifestó que el proyecto recogía 'importantes reivindicaciones históricas' del sindicato, expresó su malestar por el escaso interés del MEC para negociar aspectos referentes a las condiciones laborales y a la financiación de las universidades $[68,70]$. El Gobierno de la Generalitat de Catalunya valoró positivamente el Proyecto, pero constató que determinados aspectos del mismo no respetaban el ámbito competencial autonómico [71]. El presidente de la CRUE calificó la reforma de la LOU como 'positiva', aun siendo 'contenida' y limitada [68,72]. La Plataforma Estatal de Profesores Titulares de Escuelas Universitarias acogió con satisfacción los cambios introducidos en el texto referentes a su acceso al cuerpo de profesores titulares de universidad [73] y, por lo que hace a los profesores funcionarios, los hubo que se adhirieron a las objeciones del PP [74], pese a las denuncias que, como se comenta más adelante, se venían haciendo sobre el proceso de habilitación.

Iniciado el plazo de presentación de enmiendas al Proyecto de Ley remitido por el Gobierno, que fue ampliado repetidamente, a primeros de noviembre comparecieron ante la Comisión de Educación y Ciencia del Congreso de los Diputados diversas personalidades para informar sobre éste [75-77]: el presidente de la CRUE (quien hizo entrega de un documento con las propuestas del Consejo) [78]; el Dr. Guy Haugh, administrador principal de la Dirección General de Educación de la Unión Europea; el secretario general del Consejo Europeo de Investigación; el presidente del Consejo Económico y Social; el presidente del Colegio Oficial de Geólogos; los ex rectores de la Universidad Politécnica de Cataluña y de la Universidad Autónoma de Barcelona; el presidente del Consejo Social de la Universidad de Salamanca; seis profesores y catedráticos; el presidente de la Federación de Asociaciones de Estudiantes, y los secretarios generales de Enseñanza de los sindicatos CC.OO. y UGT. Por lo que respecta al procedimiento de selección del profesorado funcionario, la gran mayoría de los comparecientes valoraró positivamente la sustitución del sistema de habilitación por un sistema de acreditación; si bien unos pocos se mostraron disconformes con determinados aspectos del procedimiento diseñado en el Proyecto de ley. Así, los catedráticos
Francisco Sosa de la Universidad de León [76, p. 23-33] y Vicente Pedraza de la Universidad de Granada [76, p. 31-41], y el presidente del Consejo Social de la Universidad de Salamanca Salvador Sánchez-Terán [77, p. 26-33] denunciaron, como principales aspectos, la eliminación de las pruebas públicas en el sistema de acreditación, la designación directa de los miembros de las comisiones de acreditación, los peligros de una acreditación ilimitada y la indefinición sobre las características de las pruebas de acceso que deben realizar las universidades. Por otra parte, el director de escuelas empresariales de Zaragoza y el profesor de la Universidad Politécnica de Cataluña, Eusebi Jarauta, comentaron ante la Comisión parlamentaria las propuestas gubernamentales referentes a los profesores titulares de escuelas universitarias [77, p. 41-52 y 52-63].

En la sesión del pleno del Congreso de los Diputados celebrada el día 23 de noviembre, todos los grupos parlamentarios rechazaron una enmienda a la totalidad de devolución del Proyecto de Ley de modificación de la LOU presentada por el PP $[79,80]$. Y, después de la votación, se reunió la ponencia que debía elaborar el informe sobre las más de 430 enmiendas parciales al articulado presentadas por los diversos partidos; unas 60 de ellas referentes a las disposiciones sobre el profesorado funcionario [81].

Entre estas enmiendas, consideramos especialmente significativas las presentadas por parlamentarios de Convergència i Unió, del Grupo Parlamentario Vasco y del Grupo Mixto con el objetivo de acentuar el papel de las comunidades autónomas en la selección del profesorado. En ellas se proponía: que el proceso de acreditación corriera a cargo de las agencias de evaluación y acreditación de tales comunidades; que, al menos, uno de los miembros de las comisiones de acreditación perteneciera a una universidad de una comunidad autónoma en la que existiera cooficialidad lingüística; que los profesores contratados con categoría equivalente o superior a la de profesor titular de universidad pudieran solicitar acreditación como catedráticos de universidad; que la emisión del informe sobre la actividad docente e investigadora de los doctores con más de ocho años de antigüedad que aspirasen a la acreditación para catedrático de universidad fuese función exclusiva o preferente de las agencias autonómicas; que en las comisiones de acceso a los cuerpos de funcionarios docentes las univer- 
sidades se ajustaran a lo dispuesto en la normativa autonómica correspondiente, y que en las comunidades autónomas con lengua oficial propia las universidades pudieran establecer en estos concursos de acceso el requisito de conocimiento de dicha lengua. También creemos que merece mención una enmienda presentada por un parlamentario del Grupo Mixto que recogía la petición de la CRUE en el sentido de que las universidades pudieran convocar concursos de méritos entre los funcionarios de los cuerpos docentes [81].

Los días 28, 29 y 30 de noviembre, la Comisión de Educación y Ciencia del Congreso de los Diputados discutió el informe elaborado por la Ponencia, la cual había decidido no incorporar ninguna de las enmiendas presentadas [82]. Finalmente, se aprobaron 47 enmiendas de aproximación redactadas entre algunos grupos parlamentarios, una enmienda técnica y 24 enmiendas de entre las que los diversos grupos parlamentarios habían mantenido [83-85]. El dictamen de la Comisión y las enmiendas no incorporadas a éste mantenidas por los grupos parlamentarios [86] fueron discutidos y votados por el pleno del Congreso de los Diputados en una sesión celebrada el 14 de diciembre. En su intervención introductoria, la ministra Mercedes Cabrera destacó la voluntad de los distintos grupos políticos de llegar a acuerdos, 'plasmada en las enmiendas introducidas para mejorar y enriquecer la Ley, profundizando en los principios que la inspiran. El pleno aceptó una decena de enmiendas vivas después del trámite en Comisión y cuatro enmiendas transaccionales, y el texto final fue aprobado por todos los grupos parlamentarios, excepto el PP, con 189 votos afirmativos, 132 negativos y una abstención [87]. Las minorías entendieron que el proyecto había mejorado con las modificaciones incorporadas, particularmente en aspectos competenciales, pero mantuvieron discrepancias que esperaban enmendar en el Senado [88]. Por otra parte, cabe señalar que, para recibir el apoyo de los nacionalistas catalanes y vascos, fue necesario que el Gobierno retirara previamente los recursos de inconstitucionalidad contra las leyes de universidades de ambas comunidades [89].

El texto aprobado por el Congreso de los Diputados fue publicado en el Boletín Oficial de las Cortes Generales con fecha 26 de diciembre [90] $y$, al día siguiente, tuvo lugar su entrada en el Senado; se remitió a la Comisión de Educación y Ciencia, y se abrió el plazo para presentación de enmiendas [91]. El Grupo Parlamentario Popular presentó una propuesta de veto al Proyecto de Ley [92] y los diferentes grupos parlamentarios presentaron un total de 337 enmiendas a su articulado; 42 de ellas sobre aspectos relacionados con los profesores funcionarios [93]. Mientras tanto, a petición de los partidos, un grupo de 12 expertos universitarios (formado por rectores, catedráticos, investigadores y alumnos), el día 19 de febrero, acudió ante la Comisión de Educación y Ciencia para dar su opinión sobre el proyecto [94], a la vez que diversos profesores manifestaron sus críticas al Proyecto mediante la prensa [95].

Con fecha 12 de marzo, la Comisión de Educación y Ciencia del Senado rechazó con 12 votos a favor y 13 en contra el veto presentado por el Grupo Popular y, a continuación, pasó a debatir las enmiendas. Se aprobaron 14 enmiendas transaccionales (de un total de 16 elaboradas), dos enmiendas del Grupo de Senadores Nacionalistas Vascos y ocho enmiendas del Grupo Socialista no incorporadas a las transaccionales. El texto final del Dictamen se aprobó con 13 votos a favor y 12 en contra [96], y fue publicado el 16 de marzo [97]. Finalmente el Senado, el día 21 de marzo, rechazó también el veto del Grupo Parlamentario Popular y, con el voto a favor de todos los partidos excepto el del PP, aprobó el Dictamen de la Comisión previa introducción de 15 enmiendas de las que se habían mantenido vivas [98].

Las enmiendas del Senado, acompañadas de un informe motivado, se publicaron el día 29 de marzo [99], y el mismo día se produjo la ratificación definitiva de la Ley por el Congreso de los Diputados con el voto afirmativo de todos los grupos parlamentarios con la única excepción del PP [100]. El texto definitivo se publicó en el Boletín Oficial de las Cortes Generales el día 4 de abril [101] y en el Boletín Oficial del Estado el día 13 del mismo mes [102]. Unos meses más tarde, el PP recurrió ante el tribunal Constitucional varios artículos de la Ley, entre ellos los referentes a la selección del profesorado funcionario [103].

\section{Bibliografía}

1. Carreras J. Evaluación de la calidad docente y promoción del profesorado (V). Legislación universitaria españo- 
la (c): desarrollo de la Ley Orgánica de Universidades (2002-2005). Educación Médica 2007; 10: 69-85.

2. EFE. Los rectores ofrecen su apoyo al próximo Gobierno para reformar la Ley de Universidades. URL: http://www. elmundo.es/universidad/2004/04/15/actualidad.1082043. html.

3. 'La reforma de la LOU será parcial y gradual.' Comunidad Escolar, Núm. 743. 2004.

4. 'Las universidades piden que se reforme la LOU pero sin abrir un nuevo roceso constituyente". CRUE Noticias, Boletín Núm. 13 (7-V-2004). URL: htpp://www.crue.org/ boletines_crue/CRUE_Noticias_N_13.htm.

5. 'San Segundo anuncia la reforma del sistema de habilitación del profesorado y del sistema de acceso a la Universidad. Comunidad Escolar, Núm. 745. 2004.

6. Intervención de la Ministra de Educación y Ciencia, María Jesús San Segundo, en la Comisión de Educación y Ciencia del Senado (21-VI-2004). URL: http://www.mepsyd.es/ mecd/gabipren/intervenc/edu_040621_senado.pdf

7. 'Reunión de la CASUE. Conclusiones' (27-V-2004). URL: http://www.ull.es/docencia/documentos/conclusiones_ casue.htm.

8. 'Documento de la Asamblea General de la CRUE sobre cambios en la LOU y orientaciones de las políticas universitarias'. URL: htpp://www.crue.org/pdf/asambleacrue20041.pdf

9. 'Intervención de la Ministra de Educación y Ciencia, María Jesús San Segundo, en el pleno del Consejo de Coordinación Universitaria' (28-VII-2004). URL: http://www. mepsyd.es/mecd/gabipren/intervenc/edu_040728_coorduniv.pdf.

10. Pérez de Pablos S. Zapatero se compromete ante los rectores a liderar el cambio en la Universidad. El País, de 19-X-2004.

11. 'El presidente de Gobierno se compromete a liderar el impulso y cambio universitario'. Comunidad Escolar, Núm. 752. 2004.

12. 'El MEC presenta su propuesta de reforma de la LOU que pretende aprobar antes de que acabe 2005'. Comunidad Escolar, Núm. 756. 2004.

13. 'Intervención de la Ministra de Educación y Ciencia, María Jesús San Segundo, en el pleno del Consejo de Coordinación Universitaria en Valencia' (16-XII-2004). URL: http://www. mepsyd.es/mecd/gabipren/intervenc/ edu_161204_Valencia.pdf.

14. Aguirregomezcorta M. Los rectores consideran que la reforma universitaria recoge sus peticiones. El País, de 16XII-2004.

15. M.A. El PP critica el sistema de elección docente en la reforma universitaria. El País, de 18-XII-2004.

16. Agencias. Vázquez dice que el borrador de la LOU estará listo 'en pocas semanas'. URL: http://www.elmundo.es/ universidad/2005/03/09/actualidad/1110399635.html.
17. 'Declaración de la Conferencia de Rectores de las Universidades Españolas' (6-IX-2005). URL: http://www.crue.org.

18. Agencias. La nueva LOU fija un sistema de acreditación de méritos para el acceso de profesores a la Universidad. URL: http://www.elmundo.es/universidad /2005/03/09/ actualidad/1130436659.html.

19. MEC. Borrador de la Ley Orgánica de modificación de la Ley Orgánica 6/2001, de 21 de diciembre, de Universidades (27-X-2005). URL: http://www.crue.org/boletines/ boletin_N1/Adjuntos\%20de\%20AG\%2027-10-05/Borrador_Lou.pdf.

20. 'Debate sobre la reforma de la LOU'. Comunidad Escolar, Núm. 775. 2005.

21. 'La CRUE estudia el borrador de modificación de la LOU presentado por el Ministerio' (27-XII-2005). CRUE Noticias, Boletín Núm. 1 (XI-2005). URL: http://www.crue. org/boletines/boletin_N1/Boletin1.htm\#temaMes

22. Asamblea General de CASUE. Documento de conclusiones (7 y 8-XI-2005). URL: http://www.uvigo.es/goberno/ claustro/modificacion_lou/f_documentos/CASUE.pdf

23. 'Propuestas de la CRUE al borrador de modificación de la LOU. CRUE Noticias, Boletín Núm. 2 (I-2006). URL: htpp://www.crue.org/boletines/boletin_N2/Boletin 2.htm.

24. 'Acuerdo de la Asamblea General de la Conferencia de Rectores de las Universidades Españolas: modificación de la Ley Orgánica de Universidades'. Propuestas sobre Profesorado (25-I-2006). URL: http://www. crue.org/boletines/boletin_N2/adjuntos/Profesorado.pdf.

25. Morán C. Los rectores alertan de que la LOU tendrá que cambiarse otra vez en 2010. El País, de 27-I-2006.

26. García I. Los gobiernos de Cataluña y País Vasco aseguran que la LOU invade 'competencias autonómicas'. URL: http://www.elmundo.es/universidad/2005/12/14/ campus/1134579344.html.

27. Egea $\mathrm{M}^{\mathrm{a} J}$. El conseller Solà considera la LOU de 'llei cantrista i rígida. Gaceta Universitaria (14-XI-2005), p. 4.

28. Becerra J. Pilar del Castillo ex ministra de Educación, Cultura y Deporte: 'Esta reforma retrasa la modernización de la universidad'. El Mundo Campus, de 9-XI-2005.

29. FETE-UGT. Comunicado de prensa: la reforma de la LOU debe responder a las demandas de la comunidad universitaria (8-XI-2005). URL: http://www.feteugt-cyl.es/ documentos/comunicados\%20de\%20prensa/ano2005/s. universidad/Primer\%20Comunicado\%20Lou.pdf

30. FETE-UGT. Comisión Ejecutiva Federal. Propuesta de FETE-UGT al borrador de la Ley Orgánica de Modificación de la Ley Orgánica 6/2001, de 21 de diciembre, de Universidades (18-XI-2005). URL: http://www.upo.es/ ugt/docu/prop_fete_borrador_mod_lou.pdf

31. Servimedia. CCOO denuncia que la LOU lesiona al profesorado. URL: http://www.elmundo.es/universidad/ 2005/11/08/actualidad. 
32. Federación de Enseñanza de CC.OO. Plataforma de CC.OO. Modificación de la Ley Orgánica de Universidades. Valoraciones y enmiendas a la propuesta presentada por el MEC (22-XI-2005). URL: http://www.fe.ccoo.es/ universidad/38_plat_ccoo_ref_lou.pdf

33. Federación de Enseñanza de CC.OO. Análisis de la propuesta de modificación de la LOU. Comparación entre el texto actual, la propuesta del MEC y las enmiendas que ha presentado CC.OO. (15-XI-2005). URL: http://www. fe.ccoo.es/universidad/36_comp_lou_val_ccoo.pdf

34. Sección Sindical SIPU. Nota informativa (11/XI/2005). URL: http://www.api.uniovi.es/sipu/Comunicados\%20 SIPU\%20PDI/comunicado\%2011\%20de\%20noviembre\%20de\%202005.pdf

35. Servimedia. La FACU tacha de 'importante retroceso' el borrador de reforma de la LOU. URL: http://www. elmundo. es/universidad/2005/11/04/actualidad/1131110688.html.

36. ACU. Reforma de la LOU. Hoja Informativa (noviembre 2005).

37. Servimedia. Los profesores de Escuela Universitaria amenazan con ir a la huelga contra la LOU. URL: http:// www.elmundo.es/universidad/2005/11/04/actualidad/ 1131106562.html.

38. Plataforma Estatal de Profesores Titulares de Escuela Universitaria. Valoración del borrador de reforma de la LOU (4/XI/2005). URL: http://www.uv.es/infoteu/Plataforma_Estatal/Valoracion_Borrador_LOU.pdf

39. Plataforma Estatal de Profesores Titulares de Escuela Universitaria. Justificación de la reivindicación de la conversión automática de titulares de Escuela Universitaria (TEU) a titulares de universidad (TU). URL: http://www. uv.es/infoteu/razones_reivindicacion.html

40. Plataforma Estatal de Profesores Contratados que iniciaron su carrera docente bajo la LRU. Manifiesto ante el proyecto de reforma de la Ley Orgánica 6/2001, de 21 de diciembre, de Universidades (LOU) (noviembre 2005). URL: http://www.cgt-uab.net/arxiu/documents/manifiesto_estatal_contratados_lou.pdf

41. Sánchez Capelo A, presidenta de la AACTE. Carta al Director. El Mundo, de 16-III-2005.

42. Europa Press. Marcellán alerta de una posible 'diversidad' en la contratación de profesorado por las comunidades autónomas. URL: http://www. elmundo.es/universidad/2005/10/28/actualidad/1130510580.html.

43. 'Director de ANECA: la reforma de LOU no ataca los problemas de fondo'. http://actualidad.terra.es/sociedad/ artículo/director_aneca_lou_708044.htm.

44. 'ANECA: modificaciones propuestas a la LOU'. URL: http://www.universia.es/portada/actualidad/noticia_actualidad_print.jsp?noticia $=87247$.

45. 'La comunidad universitaria analiza el proyecto de reforma de la LOU'. Comunidad Escolar, Núm. 776. 2005.
46. Agencias. Ordóñez dice que no le gusta su propia reforma universitaria. El País, de 4-XI-2005.

47. 'Salvador Ordóñez defiende la reforma de la LOU por pretender aumentar la autonomía universitaria’. El País, de 13-XII-2005.

48. MEC. Nota de Prensa: Mercedes Cabrera, nueva Ministra de Educación y Ciencia (7-IV-2006). URL: http://www. mepsyd.es/multimedia/00000594.pdf.

49. 'Discurso de la Ministra de Educación y Ciencia, Mercedes Cabrera, en el acto celebrado en el Ministerio el día de su toma de posesión' (11-IV-2006). URL: http://www. mepsyd.es/mecd/gabipren/intervenc/toma_posesion_cabrera.pdf.

50. Aunión JA. El Gobierno aprobará en mayo la reforma de la ley universitaria. El País, de 21-IV-2006.

51. 'El MEC remite el proyecto de reforma de la LOU al Consejo de Coordinación Universitaria. Comunidad Escolar, Núm. 788. 2006.

52. Comisión de Educación y Ciencia. Sesión núm. 39. Cortes Generales. Diario de Sesiones del Congreso de los Diputados, Año 2006, VIII Legislatura, Núm. 586 (24-V-2006), p. 2-27.

53. MEC. Borrador del Anteproyecto de Ley Orgánica por la que se modifica la Ley Orgánica 6/2001, de 21 de diciembre, de Universidades (25-V-2006). URL: http://www. crue.org/boletines/boletin_N7/Boletin\%20N7\%20/adjuntos/Anteproyecto\%20de\%20reforma\%20de\%20la\%20 LOU (25-05-06) pdf.

54. Comisión de Educación y Ciencia. Cortes Generales. Diario de Sesiones del Senado, Año 2006, VIII Legislatura, Núm. 348 (14-VI-2006), p. 2-22.

55. 'Acuerdo de la Asamblea General de la Conferencia de Rectores de las Universidades Españolas: propuestas al borrador del Anteproyecto de Ley Orgánica por la que se Modifica la Ley Orgánica de Universidades"(29-V-2006). URL: http://www.crue.org/boletines/boletin_N7/Boletin\%20N7\%20/adjuntos/Propuestas\%20CRUE\%20al\%20 Borrador\%20Anteproyecto\%20Ley\%20reforma\%20 LOU\%20.pdf.

56. FETE-UGT. Propuesta de enmiendas al borrador del Anteproyecto de Ley Orgánica por la que se modifica la LOU (VI-2006). URL: http://fete.ugt.og/Estatal/paginas/univ/ EnmiendasLOU.pdf

57. Partido Popular. El PP le dice a Cabrera que el borrador de Ley universitaria rompe el sistema nacional docente y debilita la participación social. URL: http://www.pp.es/ index.asp? $\mathrm{p}=9528 \& \mathrm{c}=322 \mathrm{a} 45 \mathrm{a} 25 \mathrm{fb} 119 \mathrm{~b} 2 \mathrm{fac} 403 \mathrm{eba} 2$ cc11c.

58. MEC. Nota de Prensa: Mercedes Cabrera informa al Consejo de Ministros sobre el proyecto de reforma de la Ley Orgánica de Universidades (30-VI-2006). URL: http:// www.mepsyd.es/multimedia/00001455.pdf. 
59. 'El Consejo de Ministros aprueba el proyecto de reforma de la LOU'. Comunidad Escolar, Núm. 791. 2006.

60. MEC. Anteproyecto de Ley Orgánica por la que se modifica la Ley orgánica 6/2001, de 21 de diciembre, de Universidades (30-VI-2006). URL: http://www.crue.org/ boletines/boletin_N8/Boletin\%20N8/adjuntos/Aprobacion\%20LOU/Anteproyecto\%20LOU\%20consejo\%20 de\%20ministros\%2030-6-06.pdf.

61. 'Información sobre la última Asamblea General de la CRUE, celebrada el 5 de julio. CRUE Noticias, Boletín Núm. 8 (VII-2006). http://www.crue.org.boletines/boletin_N8/Boletin 8.htm.

62. Consejo de Estado. Dictamen sobre el Anteproyecto de Ley Orgánica por la que se modifica la Ley Orgánica, de 21 de diciembre, de Universidades (19-VII-2006).

63. MEC. Nota de prensa: el Consejo de Ministros aprueba el proyecto de Ley que reforma la LOU (1-IX-2006). URL: http://www.mepsyd.es/multimedia/00001752.pdf.

64. MEC. Proyecto de Ley Orgánica por la que se modifica la Ley Orgánica 6/2001, de 21 de diciembre, de Universidades (1-IX-2006). URL: http://www.crue.org/boletines/ boletin_N9/Boletin\%20No9/adjuntos/LOU\%20(Consejo\%20de\%20Ministros\%201\%20de\%20septiembre\%20 de\%202006).pdf

65. Proyecto de Ley 121/000101 Orgánica por la que se modifica la Ley Orgánica 6/2001, de 21 de diciembre, de Universidades. Boletín Oficial de las Cortes Generales. Congreso de la Diputados. VIII Legislatura, Serie A, Núm. 101-1 (8-IX-2006), p. 1-16.

66. Partido Popular. Sobre el proyecto de Ley Orgánica de Reforma de la Ley de Universidades. Pastor advierte que la reforma del Gobierno supone un retroceso en la calidad y apertura de la Universidad española (1-IX-2006). URL: http://www.pp.es/index.asp?p=10315\&c=059bbd8df7767 d3bc7829e3753c221e2

67. Morán C. La universidad pública podrá adaptarse a la LOU sin modificar sus estatutos. El Consejo de Ministros aprueba el proyecto de reforma de la educación superior. El País, de 2-IX-2006.

68. 'El Consejo de Ministros aprueba el proyecto de reforma de la LOU'. Comunidad Escolar, Núm. 793. 2006.

69. García J. El govern adverteix que la LOU envaeix competències del nou Estatut. El Punt, de 2-IX-2006.

70. Campos J. Una nueva oportunidad para el sistema educativo. El País, de 11-IX- 2006.

71. Gencat. Comunicat del Departament d'Educació i Universitats davant l'aprovació del projecte de reforma de la LOU. URL: http://www10.gencat.net/dursi/AppJava/noticies_fitxa.jsp?area $=0$ \&idioma $=0$ idnoticia $=23795$.

72. Europa Press. La conferencia de rectores aplaude la nueva ley pese a verla 'limitada'. La Vanguardia, de 4-IX-2006.

73. 'Sin plazo para ser doctor. Los profesores universitarios no tendrán un límite para obtener este título académico. Metro, de 4-IX-2006.

74. Montoro R. Catedráticos sin oposiciones. El Economista, de 4-IX-2006.

75. Comisión de Educación y Ciencia. Sesión núm. 49. Cortes Generales. Diario de Sesiones del Congreso de los Diputados, Año 2006, VIII Legislatura, Núm. 695 (6-XI-2006), p. 2-43.

76. Comisión de Educación y Ciencia. Sesión núm. 50. Cortes Generales. Diario de Sesiones del Congreso de los Diputados, Año 2006, VIII Legislatura, Núm. 698 (7-XI-2006), p. 2-63.

77. Comisión de Educación y Ciencia. Sesión núm. 51. Cortes Generales. Diario de Sesiones del Congreso de los Diputados, Año 2006, VIII Legislatura, Núm. 699 (6-XI-2006), p. 2-39.

78. 'Propuestas de la CRUE al proyecto de Ley Orgánica por la que se modifica la Ley Orgánica de Universidades'. URL: http://crue.org/boletines/boletin_N11\%20Boletin\%20N11/adjuntos/ observaciones\%20CRUE\%20ministra\%207-Nov.pdf.

79. Redacción. El parlamento rechaza la enmienda a la totalidad del PP a la reforma universitaria. El País, de 24-XI-2006.

80. Pleno y Diputación Permanente. Sesión plenaria núm. 200. Cortes Generales. Diario de Sesiones del Congreso de los Diputados, Año 2006, VIII Legislatura, Núm. 216 (23-XI-2006), p. 10988-11012.

81. Proyecto de Ley 121/000101 Orgánica por la que se modifica la Ley Orgánica 6/2001, de 21 de diciembre, de Universidades. Enmiendas e índice de enmiendas al articulado. Boletín Oficial de las Cortes Generales. Congreso de la Diputados. VIII Legislatura, Serie A, Núm. 101-9 (28-XI-2006), p. 31-185.

82. Proyecto de Ley 121/000101 Orgánica por la que se modifica la Ley Orgánica 6/2001, de 21 de diciembre, de Universidades. Informe de la ponencia. Boletín Oficial de las Cortes Generales. Congreso de la Diputados. VIII Legislatura, Serie A, Núm. 101-10 (29-XI-2006), p. 187.

83. Comisión de Educación y Ciencia. Sesión núm. 52. Cortes Generales. Diario de Sesiones del Congreso de los Diputados, Año 2006, VIII Legislatura, Núm.707 (28-XI2006), p. 2-31.

84. Comisión de Educación y Ciencia. Sesión núm 53. Cortes Generales. Diario de Sesiones del Congreso de los Diputados, Año 2006, VIII Legislatura, Núm.709 (29-XI2006), p. 2-16.

85. Comisión de Educación y Ciencia. Sesión núm. 54. Cortes Generales. Diario de Sesiones del Congreso de los Diputados, Año 2006, VIII Legislatura, Núm. 714 (30-XI-2006), p. 2-11.

86. Proyecto de Ley 121/000101 Orgánica por la que se modifica la Ley Orgánica 6/2001, de 21 de diciembre, de Universidades. Dictamen de la Comisión, escritos de man- 
tenimiento de enmiendas para su defensa ante el Pleno y voto particular. Boletín Oficial de las Cortes Generales. Congreso de la Diputados. VIII Legislatura, Serie A, Núm. 101-11 (12-XII-2006), p. 189-212.

87. Pleno y Diputación Permanente. Sesión plenaria núm. 206. Cortes Generales. Diario de Sesiones del Congreso de los Diputados, Año 2006, VIII Legislatura, Núm. 222 (14-XII-2006), p. 11239-85.

88. 'El Pleno del Congreso respalda la reforma de la LOU'. Comunidad Escolar, Núm. 800, 2006.

89. CM/AA. Las becas se ajustarán al coste de vida de cada territorio. El Congreso aprueba la reforma de la Ley de Universidades, que pasará al Senado. El País, de 15-XII-2006.

90. Proyecto de Ley 121/000101 Orgánica por la que se modifica la Ley Orgánica 6/2001, de 21 de diciembre, de Universidades. Aprobación por el Pleno. Boletín Oficial de las Cortes Generales. Congreso de la Diputados. VIII Legislatura, Serie A, Núm. 101-12 (26-XII-2006), p. 213-34.

91. Proyecto de Ley 621/000083 Orgánica por la que se modifica la Ley Orgánica 6/2001, de 21 de diciembre, de Universidades. Texto remitido por el Congreso de los Diputados. Boletín Oficial de las Cortes Generales. Senado. VIII Legislatura, Serie II, Núm. 83(a) (27-XII-2006), p. 1-26.

92. Proyecto de Ley 621/000083 Orgánica por la que se modifica la Ley Orgánica 6/2001, de 21 de diciembre, de Universidades. Propuestas de veto. Boletín Oficial de las Cortes Generales. Senado. VIII Legislatura, Serie II, Núm. 83(c) (19-II-2007), p. 29-31.

93. Proyecto de Ley 621/000083 Orgánica por la que se modifica la Ley Orgánica 6/2001, de 21 de diciembre, de Universidades. Enmiendas. Boletín Oficial de las Cortes Generales. Senado. VIII Legislatura, Serie II, Núm. 83(d) (19-II-2007), p. 33-151.

94. García I. Los expertos universitarios creen que la reforma de la LOU es 'insuficiente. El Mundo, de 21-II-2007.
95. Pla R, Dolz L, Durán A, Pastor J. La reforma de la LOU, otra oportunidad perdida. El País, de 5-III-2007.

96. Comisión de Educación y Ciencia. Cortes Generales. Diario de Sesiones del Senado, Año 2007, VIII Legislatura, Núm. 436 (12-III-2007), p. 1-29.

97. Proyecto de Ley 621/000083 Orgánica por la que se modifica la Ley Orgánica 6/2001, de 21 de diciembre, de Universidades. Dictamen de la Comisión. Boletín Oficial de las Cortes Generales. Senado. VIII Legislatura, Serie II, Núm. 83(e) (16-III-2007), p. 153-81.

98. Sesión del Pleno. Cortes Generales. Diario de Sesiones del Senado, Año 2006, VIII Legislatura, Núm. 115 (21III-2007), p. 6985-7091.

99. Proyecto de Ley 121/000101 mediante mensaje motivado al Proyecto de Ley Orgánica por la que se modifica la Ley Orgánica 6/2001, de 21 de diciembre, de Universidades. Proyecto de Ley. Boletín Oficial de las Cortes Generales. Congreso de la Diputados. VIII Legislatura, Serie A, Núm. 101-13 (29-III-2007), p. 235-88.

100. Pleno y Diputación Permanente Sesión plenaria núm. 228. Cortes Generales. Diario de Sesiones del Congreso de los Diputados, Año 2007, VIII Legislatura, Núm. 246 (29-III-2007), p.12355-412.

101. Proyecto de Ley 121/000101 Orgánica por la que se modifica la Ley Orgánica, de 21 de diciembre, de Universidades. Aprobación definitiva por el Congreso. Boletín Oficial de las Cortes Generales. Congreso de la Diputados. VIII Legislatura, Serie A, Núm. 101-14 (4-IV-2007), p. 289-314.

102. Ley Orgánica $4 / 2007$, de 12 de abril, por la que se modifica la Ley Orgánica 6/2001, de 21 de diciembre, de Universidades (B.O.E. de 13-IV-2007), p. 16241-60.

103. 'El PP recurre varios artículos de la reforma de la LOU' CRUE Noticias, Boletín Núm. 19 (VII-2007). URL: http://www.crue.org/boletines/boletin_N19/Boletin19. htm\#temaMES. 